Pacific Journal of Mathematic 


\title{
E-UNITARY COVERS FOR INVERSE SEMIGROUPS
}

\author{
D. B. McAlister and N. R. Reilly
}

An inverse semigroup is called $E$-unitary if the equations $e a=e=e^{2}$ together imply $a^{2}=a$. In a previous paper, the first author showed that every inverse semigroup has an $E$. unitary cover. That is, if $S$ is an inverse semigroup, there is an $E$. unitary inverse semigroup $P$ and an idempotent separating homomorphism of $P$ onto $S$. The purpose of this paper is to consider the problem of constructing $E$. unitary covers for $S$.

Let $S$ be an inverse semigroup and let $F$ be an inverse semigroup, with group of units $G$, containing $S$ as an inverse subsemigroup and suppose that, for each $s \in S$, there exists $g \in G$ such that $s \leqq g$. Then $\{(s, g) \in S \times G: s \leqq g\}$ is an $E$. unitary cover of $S$. The main result of $\$ 1$ shows that every $E$. unitary cover of $S$ can be obtained in this way. It follows from this that the problem of finding $E$. unitary covers for $S$ can be reduced to an embedding problem. A further corollary to this result is the fact that, if $P$ is an $E$. unitary cover of $S$ and $P$ has maximal group homomorphic image $G$, then $P$ is a subdirect product of $S$ and $G$ and so can be described in terms of $S$ and $G$ alone. The remainder of this paper is concerned with giving such a description.

1. E-unitary covers. An inverse semigroup is called $E$ unitary if the equations $e a=e=e^{2}$ together imply $a^{2}=a$. It was shown in [4] that every inverse semigroup $S$ has an $E$-unitary cover in the sense that there is an $E$-unitary inverse semigroup $P$ together with an idempotent separating homomorphism $\theta$ of $P$ onto $S$. It was further shown in [5] that every $E$-unitary inverse semigroup is isomorphic to a $P(G, \mathscr{X}, \mathscr{Y})$ where $\mathscr{X}$ is a down directed partially ordered set with $\mathscr{Y}$ an ideal and subsemilattice of $\mathscr{X}$ and where $G$ acts on $\mathscr{X}$ by order automorphisms in such a way that $\mathscr{X}=G \mathscr{Y}$; see [5] for details. The group $G$ in $P=P(G, \mathscr{X}, \mathscr{Y})$ is isomorphic to the maximum group homomorphic image $P / \sigma$ of $P$ where

$$
\sigma=\left\{(a, b) \in P \times P: e a=e b \quad \text { for some } e^{2}=e \in P\right\} .
$$

Definition 1.1. Let $S$ be an inverse semigroup and let $G$ be a group. Then an $E$-unitary inverse semigroup $P$ is an $E$-unitary cover of $S$ through $G$ if

(i) $P / \sigma \approx G$

(ii) there is an idempotent separating homomorphism $\theta$ of $P$ onto $S$. 
Thus, if $P=P(G, \mathscr{X}, \mathscr{Y})$ is an $E$-unitary cover of $S$ then $P$ is an $E$-unitary cover of $S$ through $G$. As stated, the problem of finding $E$-unitary covers of an inverse semigroup $S$ consists of finding homomorphisms onto $S$. The main result of this section shows that this problem can be replaced by an embedding problem.

Definition 1.2. [2] Let $S=S^{1}$ be an inverse semigroup, with group of units $G$. Then $S$ is a factorizable inverse semigroup if and only if, for each $a \in S$ there exists $g \in G$ such that $a \leqq g$.

Chen and Hsieh showed in [1] that every inverse semigroup $S$ can be embedded in a factorizable inverse semigroup. Indeed, let $\theta$ be a homomorphism of $S$ into the symmetric inverse semigroup $\mathscr{I}_{X}$ on a set $X$. Let $Y=X$ if $X$ is finite and $Y=X \cup X^{\prime}$, with $X \cap X^{\prime}=\square$, $|X|=\left|X^{\prime}\right|$, otherwise. Then $F=\left\{\alpha \in \mathscr{I}_{Y}: \alpha \leqq \gamma\right.$ for some permutation $\gamma$ of $Y\}$ is a factorizable inverse semigroup which contains $S \boldsymbol{\theta}$.

Proposition 1.3. Let $F$ be a factorizable inverse semigroup with group of units $G$ and let $\theta$ be a one-to-one homomorphism of an inverse semigroup $S$ into $F$. Suppose that for each $g \in G$ there exists $s \in S$ such that $s \theta \leqq g$. Then

$$
P=\{(s, g) \in S \times G: s \theta \leqq g\}
$$

is an E-unitary cover of $S$ through $G$.

Proof. It is clear that $P$ is an inverse subsemigroup of $S \times G$ and, because $F$ is factorizable, that the first projection $\pi_{1}: P \rightarrow S$ is an idempotent separating homomorphism of $P$ onto $S$. Likewise, because of the condition on the homomorphism $\theta: S \rightarrow F$, the second projection $\pi_{2}: P \rightarrow G$ is a homomorphism of $P$ onto $G$.

Now $(s, g),(t, h) \in P$ with $(s, g) \pi_{2}=(t, h) \pi_{2}$ implies $g=h$ thus $\left(s t^{-1}\right) \theta=s \theta(t \theta)^{-1} \leqq g h^{-1}=1$. It follows from this that $s t^{-1}$ is idempotent so that $e s=e t$, for some idempotent $e$ in $S$. But then $(e, 1) \in P$ and $(e, 1)(s, g)=(e, 1)(t, h)$ so that $(s, g) \sigma(t, h)$. This shows that $\pi_{2} \circ \pi_{2}^{-1} \subseteq \sigma$. On the other hand, since $\pi_{2}$ is a homomorphism onto a group, $\sigma \subseteq \pi_{2}{ }^{\circ} \pi_{2}^{-1}$. Hence $P$ has maximum group homomorphic image $G$.

Finally, since $(s, 1) \in P$ implies $s \theta \leqq 1$, and so $s^{2}=s$, if $(s, g) \pi_{2}=1$ then $(s, g)$ is idempotent. Hence $P$ is $E$-unitary.

It follows from the remarks before Proposition 1.3 that every inverse semigroup has an $E$-unitary cover. The main result of this section shows that every $E$-unitary cover of $S$ through $G$ is constructed as in Proposition 1.3 for some factorizable inverse semigroup $F$, with group of 
units $G$, containing $S$ as an inverse subsemigroup. In order to prove this, we need some lemmas.

LEMMA 1.4. Let $\theta: P(G, \mathscr{X}, \mathscr{Y}) \rightarrow S$ be an idempotent separating homomorphism of an E-unitary inverse semigroup $P(G, \mathscr{X}, \mathscr{Y})$ onto $S$. For each $A \in \mathscr{Y}$ set $N_{A}=\left\{g \in G: g^{-1} A \in \mathscr{Y}\right.$ and $\left.(A, g) \theta=(A, 1) \theta\right\}$; if $X=g A$ with $g \in G, A \in Y$, set $N_{X}=g N_{A} g^{-1}$. Then $N_{X}$ is well defined. Further, the relation $\pi$ on $\mathscr{X} \times G$ defined by

$$
(A, g) \pi(B, h) \text { if and only if } A=B \quad \text { and } g h^{-1} \in N_{A}
$$

is an equivalence on $\mathscr{X} \times G$ inducing $\theta \circ \theta^{-1}$ on $P(G, \mathscr{X}, \mathscr{Y})$.

Proof. It was shown in [5] that the subgroups $N_{A}, A \in \mathcal{Y}$ satisfy the following three conditions

(i) $N_{A} \triangleleft C_{A}=\{g \in G: g B=B$ for all $B \leqq A\}$ for $A \in \mathscr{Y}$;

(ii) $A, g A \in \mathscr{Y}$ implies $N_{g A}=g N_{A} g^{-1}$;

(iii) $A \leqq B \in \mathscr{Y}$ implies $N_{B} \subseteq N_{A}$.

We use (ii), to show that $N_{X}$ is well defined. Suppose $g A=h B, A$, $B \in \mathcal{Y}$. Then $B=h^{-1} g A$ so that, $N_{B}=\left(h^{-1} g\right) N_{A}\left(h^{-1} g\right)^{-1}$ by (ii). Thus $h N_{B} h^{-1}=g N_{A} g^{-1}$. When the $N_{X}, X \in \mathscr{X}$ are defined in this way, it is easy to see that they obey the analogs of (i), (ii), (iii) and the remainder of the lemma follows easily.

For each $C \in \mathscr{X}, g \in G$, we shall denote by $[C, g]$ the $\pi$-class containing $(C, g)$. Further, we shall denote by $\mathscr{Z}$ the set $(\mathscr{X} \times G) / \pi \cup$ G.

Lemma 1.5. For each $s \in S$ such that $s=(A, g) \theta$, set

$$
\begin{gathered}
\Delta \rho_{s}=\left\{[C, h]: h^{-1} C \leqq A\right\} \\
\text { and }[C, h] \rho_{s}=[C, h g] \text { for each }[C, h] \in \Delta \rho_{s} .
\end{gathered}
$$

Then $\rho: s \rightarrow \rho_{s}$ is a faithful representation of $S$ by one-to-one.partial transformations of $\mathscr{Z}$.

Proof. This follows straightforwardly, using the fact that $\left\{N_{X}: X \in\right.$ $\mathscr{X}$ \} obeys (i), (ii), (iii) of Lemma 1.4 .

For each $g \in G$, define $\alpha_{g}: \mathscr{Z} \rightarrow \mathscr{Z}$ by

$$
h \alpha_{g}=h g \quad \text { and } \quad[C, h] \alpha_{g}=[C, h g]
$$

for each $h \in G,[C, h] \in(\mathscr{X} \times G) / \pi$. Then, as in Lemma 1.5, it follows that $\alpha: h \rightarrow \alpha_{h}$ is a faithful representation of $G$ by permutations of $\mathscr{Z}$. Let $F=\left\{\gamma \in \mathscr{I}_{\mathscr{x}}: \gamma \leqq \alpha_{g}\right.$ for some $\left.g \in G\right\}$. 
Proposition 1.6. F is a factorizable inverse semigroup which contains $S \rho$. Further, F has group of units $\left\{\alpha_{g}: g \in G\right\}$ and for each $\alpha_{g}$ there exists $\rho_{\mathrm{s}} \leqq \alpha_{g}$; also

$$
P(G, \mathscr{X}, \mathscr{Y}) \approx\left\{(s, g) \in S \times G: s \rho \leqq \alpha_{g}\right\} .
$$

Proof. The only part requiring verification is that $P(G, \mathscr{X}, \mathscr{Y}) \approx P$ where $P=\left\{(s, g) \in S \times G: s \rho \leqq \alpha_{g}\right\}$.

Define $\phi: P(G, \mathscr{X}, \mathscr{Y}) \rightarrow P$ by $(A, g) \phi=\left((A, g) \theta, \alpha_{g}\right)$. Then, since $\theta$ is idempotent separating and $\alpha$ is faithful, $\phi$ is one-to-one. Further

$$
\begin{aligned}
(A, g) \phi(B, h) \phi & =\left((A, g) \theta, \alpha_{g}\right)\left((B, h) \theta, \alpha_{h}\right) \\
& =\left((A \wedge g B, g h) \theta, \alpha_{g} \alpha_{h}\right) \\
& =((A, g)(B, h)) \phi
\end{aligned}
$$

so $\phi$ is a homomorphism.

Finally, if $\left(s, \alpha_{g}\right) \in P$, where $s=(B, h) \theta$, then $\rho_{s} \leqq \alpha_{g}$ implies $[C, 1] \rho_{\mathrm{s}}=[C, 1] \alpha_{g}$ for each $C \leqq B$; that is, $[C, h]=[C, g]$ for each $C \leqq B$. In particular, $[B, h]=[B, g]$ so that $s=(B, h) \theta=(B, g) \theta$. Hence $\left(s, \alpha_{g}\right)=(B, g) \phi$ so that $\phi$ is onto.

Summing up, we have the following theorem.

THEOREM 1.7. Let $G$ be a group and let $S$ be an inverse semigroup. Let $F$ be a factorizable inverse semigroup with group of units $G$ which contains $S$ as an inverse subsemigroup. Suppose that, for each $g \in G$, there exists $s \in S$ such that $s \leqq g$. Then

$$
\{(s, g) \in S \times G: s \leqq g\}
$$

is an E-unitary cover of $S$ through $G$. Conversely, each E-unitary cover is isomorphic to a cover obtained in this way.

COROllary 1.8. Let $P$ be an E-unitary cover of $S$ through $G$. Then $P$ is a subdirect product of $S$ and $G$.

Theorem 1.7 shows that the problem of finding $E$-unitary covers of $S$ through $G$ is equivalent to finding an embedding of $S$ into a factorizable inverse semigroup. Such embeddings are hard to classify as they may be much larger than $S$ and $G$. On the other hand, Corollary 1.8 shows that every $E$-unitary cover of $S$ through $G$ is a subdirect product of $S$ and $G$ and therefore depends only on $S$ and $G$. In the next section, we turn to the problem of constructing those subdirect products of $S$ and $G$ which are $E$-unitary covers of $S$ through $G$. 


\section{Subdirect products of inverse semigroups.}

Definition 2.1. Let $S$ and $T$ be inverse semigroups. Then a mapping $\phi: S \rightarrow 2^{T}$ is a subhomomorphism of $S$ into $T$ if

(i) $\phi(s) \neq \square$ for each $s \in S$;

(ii) $\phi(s) \phi(t) \subseteq \phi(s t)$ for all $s, t \in S$;

(iii) $\phi\left(s^{-1}\right)=\phi(s)^{-1}$ for each $s \in S$, where, for any $A \subseteq T, A^{-1}=\left\{a^{-1}: a \in A\right\}$.

The set $\phi(S)=\{t \in T: t \in \phi(s)$ for some $s \in S\}$ is, from (ii) and (iii), an inverse subsemigroup of $T$. We say that $\phi$ is surjective if $T=\phi(S)$.

Proposition 2.2. Let $S$ and $T$ be inverse semigroups and let $\phi$ be a surjective subhomomorphism of $S$ into $T$. Then

$$
\Pi(S, T, \phi)=\{(s, t) \in S \times T: t \in \phi(s)\}
$$

is an inverse semigroup which is a subdirect product of $S$ and $T$.

Conversely, suppose that $V$ is an inverse semigroup which is a subdirect product of $S$ and $T$ and let $\psi$ be the induced homomorphism of $V$ into $S \times T$. Then $\phi$ defined by

$$
\phi(s)=\{t \in T:(s, t) \in V \psi\}
$$

is a surjective subhomomorphism of $S$ into $T$. Further

$$
V \psi=\Pi(S, T, \phi) .
$$

Proof. This is straightforward.

Proposition 2.2 shows that every $E$-unitary cover of $S$ through $G$ is determined by a subhomomorphism of $S$ into $G$; and, dually, by a subhomomorphism of $G$ into $S$. We shall consider these two approaches and the relationships between them in the later sections of the paper.

3. Subhomomorphisms into a group. In this section we shall describe the subhomomorphisms of an inverse semigroup $S$ into a group $G$. Note, however, that not every subhomomorphism $\phi$ of $S$ into $G$ gives an $E$-unitary cover of $S$ through $G$.

Definition 3.1. Let $S$ be an inverse semigroup and let $G$ be a group. Then a subhomomorphism $\phi: S \rightarrow G$ is unitary if

$$
1 \in \phi(s) \text { implies } s^{2}=s
$$


Note that, if $s$ is an idempotent then

$$
\phi(s) \phi(s)^{-1}=\phi(s) \phi\left(s^{-1}\right) \subseteq \phi\left(s s^{-1}\right)=\phi(s)
$$

so that $1 \in \phi(s)$.

Proposition 3.2. Let $S$ be an inverse semigroup and let $G$ be a group. Suppose that $\phi$ is a surjective unitary subhomomorphism of $S$ into $G$. Then $\Pi(S, G, \phi)$ is an E-unitary cover of $S$ through $G$. Conversely, let $P$ be an $E$-unitary cover of $S$ through $G$ with $\psi$ the induced homomorphism $P \rightarrow S \times G$. Then $\phi$ defined by

$$
\phi(s)=\{g \in G:(s, g) \in P \psi\}
$$

is a surjective unitary subhomomorphism of $S$ into $G$.

Proof. Suppose that $\phi$ is unitary. Then, since the idempotents of $P=\Pi(S, G, \phi)$ are the elements $(e, 1)$ with $e^{2}=e$ in $S$, the projection $\pi_{S}$ of $P$ onto $S$ is idempotent separating.

The projection $\pi_{G}: P \rightarrow G$ is onto, so, to prove that $G \approx P / \sigma$, we need only show that $\pi_{G} \circ \pi_{G}^{-1}=\sigma$. In fact, since $\pi_{G}$ is a homomorphism onto a group, so that $\sigma \subseteq \pi_{G} \circ \pi_{G}^{-1}$, we need only show that $\pi_{G} \circ \pi_{G}^{-1} \subseteq \sigma$.

Suppose that $(s, g) \pi_{G}=(t, h) \pi_{G}$ so that $g=h$. Then $(s, g)$, $(t, g) \in P$ implies $\left(s t^{-1}, 1\right) \in P$. That is, $1 \in \phi\left(s t^{-1}\right)$. Since $\phi$ is unitary, this implies $s t^{-1}$ is idempotent so that $e s=e t$ for some idempotent $e$ in $S$. But then $(e, 1)(s, g)=(e, 1)(t, g)=(e, 1)(t, h)$ so that $(s, g) \sigma(t, h)$. Thus $\pi_{G} \circ \pi_{G}^{-1} \subseteq \sigma$.

Finally, if $(s, g) \in P$ and $(e, 1)(s, g)=(e, 1)$ then $g=1$ so that $1 \in \phi(s)$. Since $\phi$ is unitary, this requires $s^{2}=s$; whence $(s, g)$ is idempotent. Hence $P$ is an $E$-unitary cover of $S$ through $G$.

Conversely, suppose that $P$ is an $E$-unitary cover of $S$ through $G$ and let $1 \in \phi(s)$. Then $(s, 1)=p \psi$ for some $p \in P$. But $1=p \psi \pi_{G}=$ $p \sigma^{\ddagger}$ implies $p^{2}=p$, since $P$ is $E$-unitary, so that $s=p \psi \pi_{s}=p \theta$, where $\theta$ is the idempotent separating homomorphism $P \rightarrow S$, is also idempotent. Hence $\phi$ is unitary.

The proof of Proposition 3.2 is strikingly reminiscent of that of Proposition 1.3. This is because Proposition 1.3 is a special case of Proposition 3.2. For, let $\theta$ be a homomorphism of $S$ into a factorizable inverse semigroup $F$, with group of units $G$, and set

$$
\phi(s)=\{g \in G: s \theta \leqq g\} .
$$

Then $g \in \phi(s), h \in \phi(t)$ implies $s \theta \leqq g, t \theta \leqq h$ so that $s \theta t \theta \leqq g h$. Thus 
$(s t) \theta \leqq g h$; that is, $g h \in \phi(s t)$. Hence $\phi(s) \phi(t) \subseteq \phi(s t)$. Further $s \theta \leqq$ $g$ if and only if $s \theta^{-1} \leqq g^{-1}$; that is $s^{-1} \theta \leqq g^{-1}$. Hence $\phi\left(s^{-1}\right)=\phi(s)^{-1}$ so that $\phi$ is a subhomomorphism. If $\theta$ is one-to-one, then clearly $\phi$ is unitary so that Proposition 1.3 follows.

In order to obtain a subhomomorphism $\phi$, as above, from a mapping $\theta: S \rightarrow F$ one need not assume that $\theta$ is a homomorphism. Only that $\theta$ is a $v$-prehomomorphism in the sense of the following definition.

Definition 3.4. Let $S, T$ be inverse semigroups then a mapping $\theta: S \rightarrow T$ is a $v$-prehomomorphism if it obeys the following two conditions.

(i) $(s t) \theta \leqq s \theta t \theta$ for each $s, t \in S$;

(ii) $\left(s^{-1}\right) \theta=(s \theta)^{-1}$ for each $s \in S$.

If $S$ and $T$ are semilattices then a $v$-prehomomorphism is just an isotone mapping of $S$ into $T$.

The results in the next lemma follow straightforwardly from the definitions.

LeMma 3.5. Let $S$ be an inverse semigroup and let $F$ be a factorizable inverse semigroup with group of units $G$. Suppose that $\theta$ is a $v$-prehomomorphism of $S$ into $F$. Then $\phi$ defined by

$$
\phi(s)=\{g \in G: s \theta \leqq g\}
$$

is a subhomomorphism of $S$ into $G$. It is surjective if and only if, for each $g \in G$, there exists $s \in S$ such that $s \theta \leqq g$; it is unitary if and only if $\theta$ is idempotent determined in the sense that a $\theta$ idempotent implies a idempotent.

Lemma 3.5 shows that $v$-prehomomorphisms of $S$ into $F$ give rise to subhomomorphisms from $S$ into $G$. On the other hand, Proposition 1.6 shows that surjective unitary subhomomorphisms of $S$ into $G$ can be obtained from embeddings of $S$ into factorizable inverse semigroups with groups of units $G$. To end this section, we show that every subhomomorphism of $S$ into $G$ is determined by a $v$-prehomomorphism $\theta$ of $S$ into a factorizable inverse semigroup $\mathscr{K}(G)$ which depends only on G.

It follows from this that every subdirect product of $S$ and $G$, in particular every $E$-unitary cover of $S$ through $G$, is determined by a $v$-prehomomorphism of $S$ into $\mathscr{K}(G)$. The problem of constructing $v$-prehomomorphisms between inverse semigroups is considered, in detail, in [6].

Let $G$ be a group. Then we shall denote by $\mathscr{K}(G)$ the set of all cosets $X=H a$ of $G$ modulo subgroups of $G$. The following simple 
lemma characterizes the members of $\mathscr{K}(G)$ among the nonempty subsets of $G$.

Lemma 3.6 [3]. Let $G$ be a group and let $X$ be a nonempty subset of $G$. Then $X \in \mathscr{K}(G)$ if and only if $X=X X^{-1} X$.

It follows from Lemma 3.6 that any nonempty intersection of cosets is again a coset. We may thus define a binary operation $*$ on $\mathscr{K}(G)$ as follows: for $X, Y \in \mathscr{K}(G)$,

$$
X * Y=\text { smallest coset that contains } X Y \text {. }
$$

Proposition 3.7 [8]. Let $G$ be a group. Then $\%(G)$ is a factorizable inverse semigroup with group of units isomorphic to $G$. The idempotents are the subgroups of $G$. Further, for $X, Y \in \mathscr{K}(G), X \leqq Y$ if and only if $X \supseteq Y$.

Proposition 3.8. Let $S$ be an inverse semigroup and let $G$ be a group. Suppose that $\phi$ is a subhomomorphism of $S$ into $G$. Then $\theta$ defined by

$$
a \theta=\phi(a) \quad \text { considered as a member of } \mathscr{K}(G)
$$

is a v-prehomomorphism of $S$ into $\mathscr{K}(G)$.

Proof. Let $X=\phi(a)$; then $X \subseteq X X^{-1} X$. On the other hand, if $g_{1}$, $g_{2}, g_{3} \in X$ then

$$
g_{1} g_{2}^{-1} g_{3} \in \phi(a) \phi(a)^{-1} \phi(a)=\phi(a) \phi\left(a^{-1}\right) \phi(a) \subseteq \phi\left(a a^{-1} a\right)=\phi(a),
$$

since $\phi$ is a subhomomorphism. Hence $X X^{-1} X \subseteq X$ and so $X=$ $X X^{-1} X$. This shows $X \in \mathscr{K}(G)$, so that $\theta$ is a mapping into $\mathscr{K}(G)$.

Next, since $\phi$ is a subhomomorphism, $a \theta b \theta \subseteq(a b) \theta$ for each $a$, $b \in S$. But $a \theta * b \theta$ is the smallest coset containing $a \theta b \theta$ so this implies $a \theta * b \theta \subseteq(a b) \theta$. That is, by Lemma 3.7, $a \theta * b \theta \geqq(a b) \theta$. Finally, again since $\phi$ is a subhomomorphism, $\left(a^{-1}\right) \theta=\phi\left(a^{-1}\right)=\phi(a)^{-1}=(a \theta)^{-1}$ for each $a \in S$. Hence $\theta$ is a $v$-prehomomorphism of $S$ into $\mathscr{K}(G)$.

It follows from Proposition 3.8 that the subdirect products of $S$ and $G$ are determined by $v$-prehomomorphisms of $S$ into $\mathscr{K}(G)$. More precisely, we have the following theorem, which sums up the results of this section. It should be pointed out however that it may be easier to find subhomomorphisms of $S$ into $G$ directly than to find $v$ prehomomorphisms of $S$ into $\mathscr{K}(G)$. 
(A). Let $\theta$ be a $v$-prehomomorphism of $S$ into $\mathscr{K}(G)$ and suppose that, for each $g \in G$, there exists $s \in S$ such that $s \theta \leqq\{g\}$; i.e. $g \in s \theta$. Then $\{(s, g): g \in s \theta\}$ is an inverse semigroup which is a subdirect product of $S$ and $G$. Every subdirect product of $S$ and $G$ is of this form for some $v$-prehomomorphism of $S$ into $\mathscr{K}(G)$.

(B). With $\theta$ as in (A), $\{(s, g): g \in s \theta\}$ is an E-unitary cover of $S$ through $G$ if and only if $\theta$ is idempotent determined. Every E-unitary cover of $S$ through $G$ is of this form for some idempotent determined $v$-prehomomorphism of $S$ into $\mathscr{K}(G)$.

4. Subhomomorphisms from a group. In $\S 3$, we characterized the $E$-unitary covers of an inverse semigroup $S$, through a group $G$, as subdirect products $\Pi(S, G, \phi)$ with $\phi$ a subhomomorphism of $S$ into $G$. They can also be described in the form $\Pi(G, S, \phi)$ with $\phi$ a subhomomorphism of $G$ into $S$. In this section, we give such a description. As might be expected the results obtained are, in a sense, dual to those in $\$ 3$.

Definition 4.1. Let $S$ and $T$ be inverse semigroups. Then a mapping $\theta: S \rightarrow T$ is a $\wedge$-prehomomorphism of $S$ into $T$ if it obeys the following two conditions.

(i) $a \theta b \theta \leqq(a b) \theta$ for each $a, b \in S$;

(ii) $\left(a^{-1}\right) \theta=(a \theta)^{-1}$ for each $a \in S$.

Proposition 4.2. Let $S$ be an inverse semigroup and let $G$ be a group. Suppose that $T$ is an inverse semigroup containing $S$ and let $\theta$ be $a$ $\wedge$-prehomomorphism of $G$ into $T$. Then $\phi$ defined by

$$
\phi(g)=\{s \in S: s \leqq g \theta\}
$$

is a subhomomorphism of $G$ into $S ; \phi$ is surjective if and only if, for each $s \in S$ there exists $g \in G$ such that $s \leqq g \theta$.

The semigroup $\Pi(G, S, \phi)$ is E-unitary. It is an $E$-unitary cover of $S$ through $G$ if $\phi$ is surjective.

Proof. The fact that $\phi$ is a subhomomorphism and the statement about the surjectivity of $\phi$ are readily verified.

Let $a=1 \theta$; then, since $\theta$ is a $\wedge$-prehomomorphism

$$
a=a a^{-1} a=a a a \leqq(1.1) \theta a=a^{2} \leqq a
$$

so that $a$ is idempotent. Let $(g, s) \in \Pi(G, S, \phi)$ and suppose that $(g, s)(1, e)=(1, e)$ where $e$ is idempotent. Then $g=1$ so that $s \leqq 1 \theta=$ $a$; thus $s$ is idempotent. Hence $\Pi(G, S, \phi)$ is $E$-unitary. 
Suppose that $\phi$ is surjective. Then, if we identify $S \times G$ with $G \times S, \Pi(G, S, \phi)=\Pi\left(S, G, \phi^{*}\right)$ where $g \in \phi^{*}(s)$ if and only if $s \in$ $\phi(g)$. Since $1 \in \phi^{*}(s)$ implies $s \leqq 1 \theta=a$, which is idempotent, $\phi^{*}$ is unitary. Hence, by Proposition $3.2, \Pi(G, S, \phi)$ is an $E$-unitary cover of $S$ through $G$.

Proposition 4.2 is analogous to Proposition 3.2. The next proposition is similar to Proposition 3.8; it shows that every $E$-unitary cover of $S$ through $G$ is determined by a $\wedge$-prehomomorphism of $G$ into a semigroup $C(S)$ depending only on $S$.

Definition 4.3 [9]. Let $S$ be an inverse semigroup. Then a nonempty subset $H$ of $S$ is called permissible if

(i) $a \in H, b \leqq a$ implies $b \in H$;

(ii) $a, b \in H$ implies $a b^{-1}, a^{-1} b$ idempotent.

Schein [9] shows that the set $C(S)$ of permissible subsets of $S$ forms an inverse semigroup under subset multiplication. Further $S$ can be embedded in $C(S)$ by means of the homomorphism $\eta$ given by

$$
a \eta=\{x \in S: x \leqq a\}
$$

for each $a \in S$.

Proposition 4.4. Let $S$ be an inverse semigroup and let $G$ be a group. Suppose that $\phi$ is a surjective subhomomorphism of $G$ into $S$ such that $\Pi(G, S, \phi)$ is an E-unitary cover of $S$ through $G$. Then $\phi(g)$ is permissible for each $g \in G$ and $\theta$ defined by

$$
g \theta=\phi(g) \quad \text { considered as a member of } C(S)
$$

is a $\wedge$-prehomomorphism of $G$ into $C(S)$. Further

$$
\Pi(G, S, \phi)=\{(g, s) \in G \times S: s \leqq g \theta\} ;
$$

here we identify $S$ with $S \eta$.

Proof. Suppose $a \in \phi(g), b \leqq a$; thus $b=e a$ for some $e^{2}=$ $e \in S$. Then $(g, a) \in P=\Pi(G, S, \phi)$ and $(1, e) \in P$ so that $(1, e)(g, a)=$ $(g, b) \in P$. Hence $b \in \phi(g)$. Next, suppose $a, c \in \phi(g)$ then $(g, a)$, $(g, c) \in P$ so that $\left(1, a^{-1} c\right) \in P$. Since $P$ is an $E$-unitary cover of $S$ through $G$, with $\pi_{G} \circ \pi_{G}^{-1}=\sigma$, where $\pi_{G}$ denotes the projection of $P$ onto $G$, this implies that $a^{-1} c$ is an idempotent. Similarly $a c^{-1}$ is an idempotent. Hence $\phi(g)$ is permissible.

It is now easy to show that $\theta$ is a $\wedge$-prehomomorphism and, because $X \leqq Y$ in $C(S)$ if and only if $X \subseteq Y$, that $P=\{(g, s) \in G \times S: s \leqq g \theta\}$. 
If we combine the results of Propositions 4.2 and 4.4, then we obtain the following dual to Theorem 3.9.

THEOREM 4.5. Let $S$ be an inverse semigroup and let $G$ be a group. Let $\theta$ be a $\wedge$-prehomomorphism of $G$ into $C(S)$ such that, for each $s \in S$ there exists $g \in G$ with $s \leqq g \theta$. Then

$$
\{(g, s) \in G \times S: s \leqq g \theta\}
$$

is an E-unitary cover of $S$ through $G$. Conversely, each E-unitary cover of $S$ through $G$ has this form for some $\wedge$-prehomomorphism $\theta$ of $G$ into $C(S)$.

\section{Examples}

5.1. Free group covers. Let $S$ be an inverse semigroup and let $X$ be a set of generators for $S$ as an inverse semigroup. Let $X^{-1}$ be a set in one to one correspondence with, but disjoint from, $X$. Then there is a homomorphism $\theta$ from the free semigroup $F_{X \cup X^{-1}}$, on $X \cup X^{-1}$, onto $S$ such that $x \theta^{-1}=x^{-1} \theta$ for each $x \in X$. Similarly, there is a homomorphism $\psi: F_{X \cup X^{-1}} \rightarrow F G_{X}$, the free group on $X$ such that $x \psi^{-1}=x^{-1} \psi$ for each $x \in X$. Define $\phi: S \rightarrow 2^{F G_{x}}$ by

$w \in \phi(s)$ if and only if $w=u \psi$ for some $u \in F_{X \cup X^{-1}}$

with $u \theta=s$; that is $\phi(s)=s \theta^{-1} \psi$ for some $s \in S$.

Proposition 5.1. $\phi$ is a surjective unitary subhomomorphism of $S$ into $F G_{X}$.

Proof. It is straightforward to show that $\phi$ is a surjective subhomomorphism of $S$ into $F G_{X}$. Suppose that $1 \in \phi(s)$. Then there exists $w \in F_{X \cup X^{-1}}$ such that $w \theta=s, w \psi=1$.

Let $\eta$ be the canonical homomorphism from $F_{X \cup X^{-1}}$ into the free inverse semigroup $F I_{X}$ on $S$. Then both $\theta$ and $\psi$ can be factored through $\eta$. Since $w \psi=1$ and $F I_{X}$ is $E$-unitary [7] it follows that $w$, regarded as an element of $F I_{X}$, is idempotent. Hence $s=w \theta$ is an idempotent of $S$. This shows that $\phi$ is unitary.

As a result of the freeness of $F G_{X}, \Pi\left(S, F G_{X}, \phi\right)$, with $\phi$ as above, has a weak universal property.

Proposition 5.2. Let $S$ be an inverse semigroup and let $P=$ $\Pi\left(S, F G_{X}, \phi\right)$ as above with $\alpha$ the homomorphism $P \rightarrow S$. Suppose that $Q$ is an E-unitary cover of $S$ through $G$ with homomorphism $\beta: Q \rightarrow S$. Then there is a homomorphism $\gamma: P \rightarrow Q$ such that $\alpha=\gamma \beta$. 
Proof. With the notation above, we have the following diagram of maps

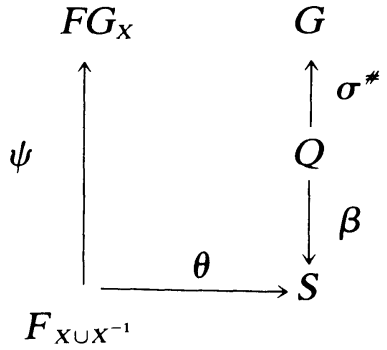

For each $x \in X$, choose $y \in Q$ such that $y \beta=x \theta$. Then there is a homomorphism $\nu: F_{X \cup X^{-1}} \rightarrow Q$ such that $x \nu \beta=x \theta$ and $(x \nu)^{-1}=x^{-1} \nu$ for each $x \in X$. Then $\nu \sigma^{4}$ is a homomorphism of $F_{X \cup X^{-1}}$ into $G$ and can be factored through $\psi$. That is, there is a homomorphism $\delta: F G_{X} \rightarrow G$ such that $\nu \sigma^{\sharp}=\psi \delta$.

From the definition of $P, P=\left\{(w \theta, w \psi): w \in F_{X \cup X^{-1}}\right\}$. Define $\gamma: P \rightarrow Q$ by $(w \theta, w \psi) \gamma=w \nu$. Then $w \theta=u \theta, w \psi=u \psi$ implies $w \psi \delta=$ $u \psi \delta$, that is $w \nu \sigma^{\natural}=u \nu \sigma^{\natural}$ and $w \nu \beta=u \nu \beta$. Since $Q$ is an $E$-unitary cover of $S$ through $G$, Corollary 1.8 shows that $u \nu=w \nu$. Hence $\gamma$ is well defined; it is clearly a homomorphism. Further, from the definition,

$$
(w \theta, w \psi) \gamma \beta=w \nu \beta=w \theta=(w \theta, w \psi) \alpha
$$

for each $(w \theta, w \psi) \in P$. Hence $\alpha=\gamma \beta$.

5.2. The Preston-Vagner cover. Let $\rho: S \rightarrow \Phi_{S}$ be the Preston-Vagner representation of an inverse semigroup $S$ and let $Y=S$ if $S$ is finite, if not $Y=S \cup S^{\prime}$ with $S \cap S^{\prime}=\square,|S|=\left|S^{\prime}\right|$. Then $F=\left\{\alpha \in \mathscr{I}_{Y}: \alpha \leqq \gamma\right.$ for some permutation $\gamma$ of $\left.Y\right\}$ is a factorizable inverse semigroup containing $S \rho$. It gives rise to the subhomomorphism $\phi$ where, for each $s \in S$,

$$
\begin{aligned}
\phi(s) & =\left\{\alpha: \rho_{s} \leqq \alpha, \alpha \text { a permutation of } Y\right\} \\
& =\left\{\alpha: x \alpha=x s \text { for each } x \in S s s^{-1}, \alpha \text { a permutation of } Y\right\}
\end{aligned}
$$

This subhomomorphism gives an $E$-unitary cover of $S$ through $K$ where

$$
K=\left\{\alpha \in S_{Y}:(x e) \alpha=x(e \alpha) \text { for all } x \in S \text { and some } e^{2}=e \in S\right\},
$$

where $S_{Y}$ denotes the symmetric group on $Y$. 
5.3. E-unitary covers of bisimple inverse semigroups. A construction is given in [6] for the $v$-prehomomorphisms $\theta$ of a bisimple inverse semigroup $S$ into an inverse semigroup $T$. When applied to the semigroup $\mathscr{K}(G)$ of cosets of a group $G$, this construction specializes to give the following construction for the $E$-unitary covers of $S$ through $G$.

Let $H$ be a subgroup of $G$ and let $S(H)=\left\{a \in G: a H a^{-1} \subseteq\right.$ $H$. Then $S(H)$ is a subsemigroup of $G$ and $(G / H, S(H) / H)$ is a partial semigroup under the multiplication $*$ :

$$
X * Y=X Y \quad \text { for each } \quad X \in S(H) / H, \quad Y \in G / H .
$$

Pick an idempotent $e \in S$ and set $R_{e}=\left\{a \in S: a a^{-1}=e\right\}, P_{e}=R_{e} \cap e S e$ and let $\theta: R_{e} \rightarrow G / H$ be a one-to-one mapping such that the following hold

(i) $\quad a \theta \in S(H) / H$ if $a \in P_{e}$

(ii) $a \theta b \theta=(a b) \theta$ for $a \in P_{e}, b \in R_{e}$

(iii) $G=\cup\left\{a \theta^{-1} b \theta: a, b \in R_{e}\right\}$.

Then $\left\{(s, g) \in S \times G: g \in a \theta^{-1} b \theta\right.$ where $\left.s=a^{-1} b\right\}$ is an $E$-unitary cover of $S$ through $G$. Conversely, each such has this form for some $\theta: R_{e} \rightarrow G / H$ as above.

5.4. E-unitary covers for semilattices of groups. A construction is given in [6] for the $v$-prehomomorphisms $\theta$ of a semilattice of groups $S$ into an inverse semigroup $T$. When applied to the semigroup $\mathscr{K}(G)$ of cosets of a group $G$, this construction specializes to give the following description of the $E$-unitary covers of $S$ through $G$.

Let $E$ be a semilattice and let $\theta$ be an anti-isotone mapping of $E$ into the lattice of subgroups of $G$. For each $e \in E$ set $G_{e}=e \theta$ and $C_{e}=\left\{a \in G: a G_{f} a^{-1}=G_{f}\right.$ for each $\left.f \leqq e\right\}$. Then $G_{e}$ is a normal subgroup of $C_{e}$ and the groups $K_{e}=C_{e} / G_{e}$ form a semilattice of groups $S L(E, \theta, \mathscr{K}(G))$ with linking homomorphisms $\phi_{e, f}: K_{e} \rightarrow K_{f}$ given by

$$
X \phi_{e, f}=G_{f} X \quad \text { for each } \quad X \in K_{e}, \quad e \geqq f .
$$

Suppose that $S$ is a semilattice of groups with semilattice of idempotents $E$. Suppose that $\theta$ is an anti-isotone mapping of $E$ into the lattice of subgroups of $G$ and let $\phi$ be an idempotent determined homomorphism of $S$ into $S L(E, \theta, \mathscr{K}(G))$ such that $G=$ $\cup\{a \phi: a \in S\}$. Then

$$
\{(s, g) \in S \times G: g \in s \phi\}
$$

is an $E$-unitary cover of $S$ through $G$. Conversely, each such has this form for some $\theta: E \rightarrow \mathscr{K}(G)$ and $\phi: S \rightarrow S L(E, \theta, \mathscr{K}(G))$. 


\section{REFERENCES}

1. S. Y. Chen and S. C. Hsieh, Factorizable inverse semigroups, Semigroup Forum, 8 (1974), 283-297.

2. A. H. Clifford and G. B. Preston, Algebraic theory of semigroups, Vols. 1 and 2. Math. Surveys of the American Math. Soc. (Providence, R. I., Vol. 1 (1961), Vol. 2 (1967)).

3. P. Dubreil, Contribution à la théorie des demi-groupes, Mèm. Acad. Sci. Inst. France, 63 (1941), $1-52$.

4. D. B. McAlister, Groups, semilattices and inverse semigroups, Trans. Amer. Math. Soc., 192 (1974), 227-244.

5. - Groups, semilattices and inverse semigroups, II, Trans. Amer. Math. Soc., 196 (1974), 351-370.

6. - $\quad$-Prehomomorphisms on inverse semigroups.

7. H. E. Scheiblich, Free inverse semigroups, Proc. Amer. Math. Soc., 38 (1973), 1-7.

8. B. M. Schein, Semigroups of strong subsets (Russian), Volžskiī Matem. Sbornik, Kuỉbyšev, 4 (1966), 180-186.

9. Completions, translational hulls and ideal extensions of inverse semigroups, Czechoslovak Math. J., 23 (1973), 575-609.

Received May 24, 1976. This research was partly supported by a grant from the National Science Foundation and a grant from the National Research Council.

NORTHERN ILLINOIS UNIVERSITY

DEKALB, IL 60115

AND

Simon Fraser University

Burnaby, British Columbia, Canada 



\section{Pacific Journal of Mathematics}

Vol. 68, No. 1

March, 1977

Richard Julian Bagby, On $L^{p}, L^{q}$ multipliers of Fourier transforms . .......

Robert Beauwens and Jean-Jacques Van Binnebeek, Convergence theorems in

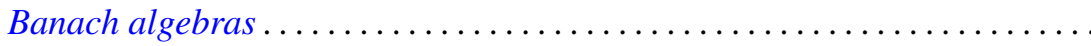

James Cyril Becker, Skew linear vector fields on spheres in the stable

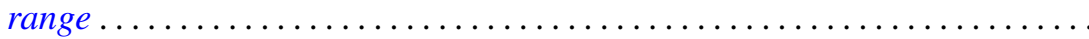

Michael James Beeson, Continuity and comprehension in intuitionistic formal

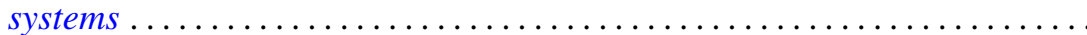

James K. Deveney, Generalized primitive elements for transcendental field

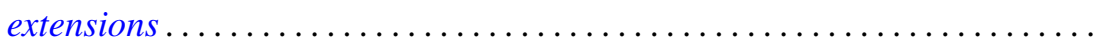

Samuel S. Feder, Samuel Carlos Gitler and K. Y. Lam, Composition properties

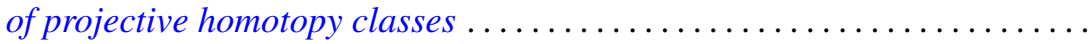

Nathan Jacob Fine, Tensor products of function rings under composition ......

Benno Fuchssteiner, Iterations and fixpoints . . . . . . . . . . . . . .

Wolfgang H. Heil, On punctured balls in manifolds

Shigeru Itoh, A random fixed point theorem for a multivalued contraction

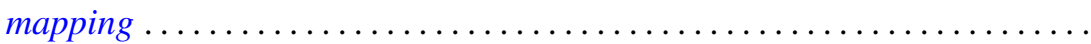

Nicolas P. Jewell, Continuity of module and higher derivations . . . . . . ......

Roger Dale Konyndyk, Residually central wreath products . . . . . . . . . . .

Linda M. Lesniak and John A. Roberts, On Ramsey theory and graphical

parameters.

Vo Thanh Liem, Some cellular subsets of the spheres.

Dieter Lutz, A perturbation theorem for spectral operators

P. H. Maserick, Moments of measures on convex bodies ... . . .

Stephen Joseph McAdam, Unmixed 2-dimensional local domains . .

D. B. McAlister and Norman R. Reilly, E-unitary covers for inverse semigroups...

William H. Meeks, III and Julie Patrusky, Representing codimension-one

homology classes by embedded submanifolds . . .

Premalata Mohapatro, Generalised quasi-Nörlund summability . .

Takahiko Nakazi, Superalgebras of weak-*Dirichlet algebras .

Catherine Louise Olsen, Norms of compact perturbations of operators .

William Henry Ruckle, Absolutely divergent series and isomorphism of

subspaces. II.

Bernard Russo, On the Hausdorff-Young theorem for integral operators .

Arthur Argyle Sagle and J. R. Schumi, Anti-commutative algebras and

homogeneous spaces with multiplications ............

Robert Evert Stong, Stiefel-Whitney classes of manifolds .

D. Suryanarayana, On a theorem of Apostol concerning Möbius functions of

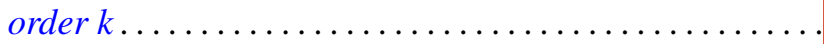

Yoshio Tanaka, On closedness of $C$ - and $C^{*}$-embeddings . . 